\title{
Influência do Primeiro Desbaste na Morfometria de Tectona grandis L.f. em Roraima
}

\author{
Luiz Fernandes Silva Dionisio ${ }^{1}$, Hilton Xavier de Araújo ${ }^{2}$, \\ Ruy Guilherme Correia ${ }^{1}$, Walmer Bruno Rocha Martins ${ }^{1}$, \\ Jéssica Saraiva da Costa ${ }^{1}$, Francisco Clemilto da Silva Maciel $^{3}$
}

\begin{abstract}
${ }^{1}$ Programa Pós-graduação em Ciências florestais, Universidade Federal Rural da Amazônia - UFRA, Belém/PA, Brasil ${ }^{2}$ Programa Pós-graduação em Agronomia, Universidade Federal de Roraima - UFRR, Boa Vista/RR, Brasil ${ }^{3}$ Instituto Federal de Roraima - UFRR, Boa Vista/RR, Brasil
\end{abstract}

\begin{abstract}
RESUMO
Objetivou-se com este trabalho avaliar o efeito do primeiro desbaste sobre a morfometria de um plantio de Tectona grandis L.f. (teca). O delineamento experimental foi o de blocos ao acaso, com quatro tratamentos e três repetições sendo: T1 = testemunha (sem desbaste), T2, T3 e T4 = remoção da área basal de 20\%, 30\% e 40\% respectivamente. As variáveis morfométricas avaliadas foram: proporção de copa (PC), índice de abrangência (IA), grau de esbeltez (GE), índice de saliência (IS), formal de copa (FC) e comprimento de copa (CC). A aplicação de desbastes em teca influenciou significativamente em todas as variáveis morfométricas avaliadas, com exceção de proporção de copa (PC) e índice de saliência (IS). As intensidades de desbastes (20\%, 30\% e 40\%) não apresentaram diferença significativa para as variáveis: altura comercial do fuste (HF), comprimento de copa (CC), diâmetro de copa (DC), proporção de copa (PC) e forma de copa (FC) da Tectona grandis até o quarto ano pós-desbaste.
\end{abstract}

Palavras-chave: Amazônia, teca, manejo florestal.

\section{Influence of First Thinning in Morphometry of Tectona grandis L.f. on Roraima}

\begin{abstract}
The aim of this study was to evaluate the effect of first thinning on the morphometry of trees in a forest plantation of Tectona grandis Lf (teak). The experimental design used was randomized block with four treatments and three replications were: $\mathrm{T} 1=$ control treatment (no thinning), $\mathrm{T} 2, \mathrm{~T} 3$ end $\mathrm{T} 4$ with the removal of basal area the 20,30 and $40 \%$ respectively. The evaluated morphometric variables were: crown proportion $(\mathrm{PC})$, coverage index (AI), slenderness degree (GE), salience index (IS), crown form (FC) and crown length (CC). The application of thinning in teak, significantly influenced in all morphometric variables analyzed, except for the proportion of room (PC) and the projection index (SI). The thinning intensities $(20 \%, 30 \%$ and $40 \%)$ presented no significant differences for variables: commercial bole height (HF), crown length $(\mathrm{CL})$, crown diameter (DC), crown proportion (PC) and crown form (FC) of teak to the fourth post-thinning year.
\end{abstract}

Keywords: Amazon, teak, management forestry. 


\section{INTRODUÇÃO}

A demanda por matéria-prima de qualidade para o abastecimento da indústria madeireira está cada vez maior (Weber et al., 2013), com aumento expressivo do setor brasileiro de florestas plantadas. Atualmente, mais do que aumentar a área plantada, há preocupação em melhorar a produtividade das plantações (Medeiros et al., 2015).

Além dos fatores edafoclimáticos favoráveis para a silvicultura, novas tecnologias estão sendo utilizadas, destacando-se os testes de progênies e a seleção assistida de ideótipos por marcadores moleculares (Schuhli \& Paludzyszyn, 2010). Esse aprimoramento leva o Brasil a se destacar na produtividade florestal, tanto de coníferas como de folhosas.

A teca (Tectona grandis L.f. - Lamiaceae) é uma espécie arbórea de rápido crescimento, originária do sul e sudeste asiático que vem se mostrando promissora em plantios comerciais, principalmente pelas ótimas característica da madeira (Figueiredo et al., 2005; Ângelo et al., 2009). Além disso, a teca também apresenta como vantagens a resistência a pragas, doenças (Motta et al., 2013) e incêndios florestais (Figueiredo, 2001).

Os primeiros plantios de teca no Brasil ocorreram na década de 1960, implantados pela empresa Cáceres Florestal S.A., no Estado do Mato Grasso (Caldeira \& Oliveira, 2008; Costa, 2011). Atualmente, a espécie vem sendo cultivada no Pará, Acre, Rondônia, Mato Grosso (Miranda, 2013) e Roraima.

Os tratamentos silviculturais, dentre eles desbastes de condução, são considerados essenciais para promover maior produtividade e qualidade às árvores remanescentes (Santos et al., 2015). No entanto, os desbastes em povoamentos de teca são efetuados em função do crescimento em volume e, principalmente, de área basal (Caldeira \& Oliveira, 2008; Pelissari et al., 2013), dando-se pouca ou nenhuma relevância à morfometria dessa espécie, sobretudo pós-desbaste.

O estudo da morfometria e das relações morfométricas de espécies florestais é importante para determinar o grau de concorrência de um povoamento, assim como a estabilidade, a produção de cada indivíduo no sítio e até mesmo o período considerado adequado ecologicamente para a realização dos desbastes (Durlo \& Denardi, 1998; Durlo et al., 2004; Santos et al., 2015). O efeito dos desbastes nas relações morfométricas das árvores é confirmado por vários estudos (Orellana \& Koehler, 2008; Tonini et al., 2008; Roman et al., 2009; Condé et al., 2013, 2014; Figueiredo et al., 2014).

A copa é um órgão vital das árvores e está normalmente correlacionada com o seu crescimento em diâmetro (Silveira et al., 2015) e altura (Padoin \& Finger, 2010; Santos et al., 2014; Wink et al., 2012). Mas as características naturais de desenvolvimento da copa podem sofrer modificações de acordo com a idade e as condições edafoclimáticas do sítio (Sanquetta et al., 2014).

Outro fator determinante é o desbaste, pois ele proporciona mais espaço para expansão da copa e altura e, consequentemente, diâmetro dos indivíduos remanescentes, dependendo da sua intensidade, não havendo estudos sobre o seu efeito na morfometria da teca. Nesse sentido, o objetivo deste trabalho foi avaliar o efeito da intensidade do primeiro desbaste sobre a morfometria de Tectona grandis L.f. (teca), no município de Iracema, centro-oeste de Roraima.

\section{MATERIAL E MÉTODOS}

O estudo foi desenvolvido em um povoamento equiâneo e homogêneo de teca, com 200 hectares implantados em 2001, sendo que a área do presente trabalho corresponde a 20 hectares, com espaçamento inicial de $3 \times 2 \mathrm{~m}$, pertencente à empresa Madeireira Mendes Ross Ltda., localizada no município de Iracema, no estado de Roraima (Figura 1), a $85 \mathrm{~km}$ da capital Boa Vista.

No plantio, foram utilizadas mudas do tipo "toco", produzidas a partir de sementes oriundas do município de Cáceres, Mato Grosso. Antes do plantio, a área foi utilizada para pastagem e o preparo do solo constituiu apenas de uma gradagem, sem adubação de plantio e de manutenção.

O clima da região é do tipo Awi, segundo a classificação de Köppen, caracterizado como quente e úmido, com estações de chuva e de seca bem definidas. A precipitação média anual no campo experimental Serra da Prata da Embrapa, a 30 km, aproximadamente, da área de estudo, é de $1.844 \mathrm{~mm}$, com os meses mais chuvosos de maio a julho (Mourão et al., 2003). Os solos predominantes na região pertencem às classes Argissolo e Latossolo (Santos et al., 2006). Nas Tabelas 1 e 2 são 


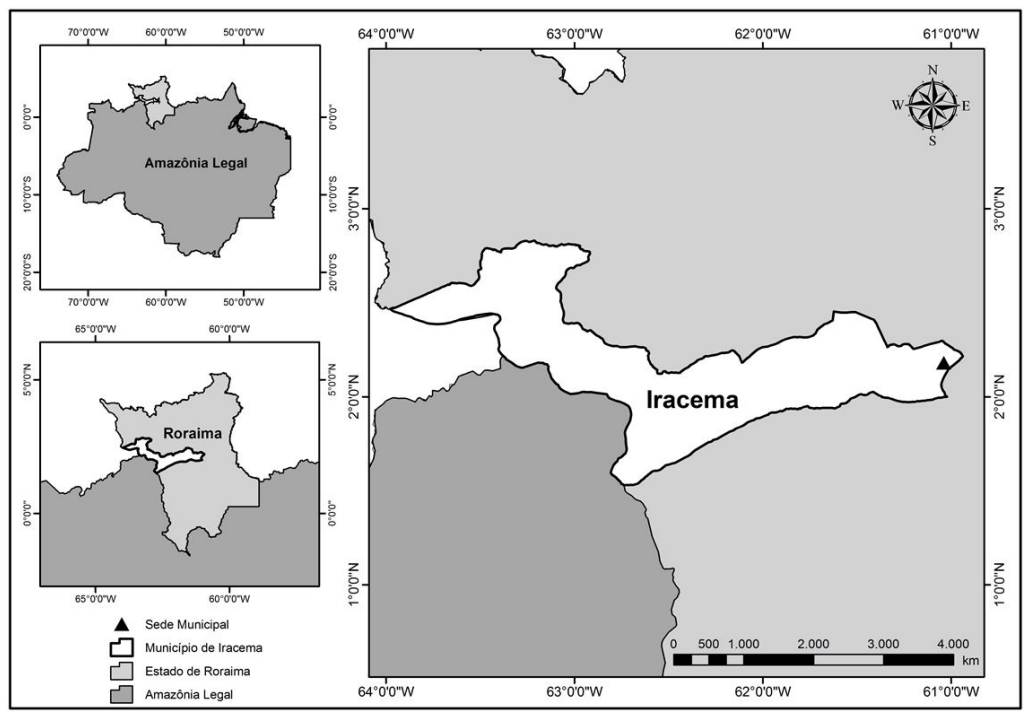

Figura 1. Localização da região do experimento no município de Iracema, Estado de Roraima.

Figure 1. Location of the experiment region in the Iracema city, Roraima state.

Tabela 1. Atributos químicos em duas profundidades do solo do sítio experimental.

Table 1. Chemical attributes in two soil depths of the experimental site.

\begin{tabular}{|c|c|c|c|c|c|c|c|c|c|}
\hline Prof. & pH & M.O. & $\mathbf{P}$ & $\mathrm{Ca}$ & Mg & $\mathbf{K}$ & $\mathbf{T}$ & $\mathbf{V}$ & m \\
\hline$(\mathrm{cm})$ & $\left(\mathrm{H}_{2} \mathrm{O}\right)$ & g. kg ${ }^{-1}$ & $\mathrm{mg} \cdot \mathrm{dm}^{-3}$ & \multicolumn{4}{|c|}{$\mathrm{cmol}_{\mathrm{c}} \cdot \mathrm{dm}^{-3}$} & \multicolumn{2}{|c|}{$\%$} \\
\hline $0-20$ & 5,8 & 18,9 & 1,1 & 1,9 & 0,5 & 0,01 & 3,0 & 52 & 1 \\
\hline $20-40$ & 5,5 & 12,8 & 0,7 & 1,1 & 0,4 & 0,01 & 3,7 & 39 & 4 \\
\hline
\end{tabular}

$\mathrm{pH}_{\mathrm{H} 2 \mathrm{O}}$; M.O. (matéria orgânica): método colorimétrico; P, K: Mehlich 1; Ca e Mg: método Kcl 1M; T = CTA potencial; V: saturação por bases; $\mathrm{m}=$ índice de saturação por bases.

Tabela 2. Caracterização física em duas profundidades do solo do sítio experimental.

Table 2. Physical characterization in two soil depths of the experimental site.

\begin{tabular}{rccc} 
Prof. & Areia & Silte & Argila \\
\cline { 2 - 4 }$(\mathbf{c m})$ & & $\%$ & \\
$0-20$ & 61 & 11 & 28 \\
$20-40$ & 63 & 11 & 26 \\
\hline
\end{tabular}

Método de densímetro.

apresentadas a caracterização física e química (textura) em duas profundidades do solo na área experimental.

O delineamento experimental utilizado foi em blocos ao acaso com três repetições e quatro tratamentos: $\mathrm{T} 1=0 \%$ (sem desbaste); $\mathrm{T} 2$ = remoção de 20\%; T3 = de 30\%; T4 = de 40\% da área basal. Foram instaladas parcelas retangulares de 30 metros de comprimento por $28 \mathrm{~m}$ de largura, com 10 linhas com 14 plantas. Foram consideradas duas linhas de bordadura para evitar o efeito de externo, restando 6 linhas com 10 plantas na área útil. Todas as árvores dentro da parcela útil foram numeradas e marcadas com tinta.

O desbaste do tipo seletivo por baixo foi realizado em junho de 2007, seis anos após o plantio. Os critérios para a seleção das árvores a serem desbastadas consideraram a seguinte ordem: estado fitossanitário, altura total do fuste, forma e qualidade do fuste e árvores com menores valores de diâmetro a 1,3 m de altura.

A medição foi realizada quatro anos após o desbaste (junho de 2011), quando fora medidos o DAP (1,30 m de altura, com fita métrica), a altura total, comprimento e diâmetro de copa (nas direções norte-sul e leste-oeste), com hipsômetro Vertex III. A partir dessas variáveis, calcularam-se as relações morfométricas, de acordo com a Figura 2.

Os pressupostos para a realização da análise de variância (Homogeneidade e Normalidade) foram avaliados utilizando-se os testes de Levene e 


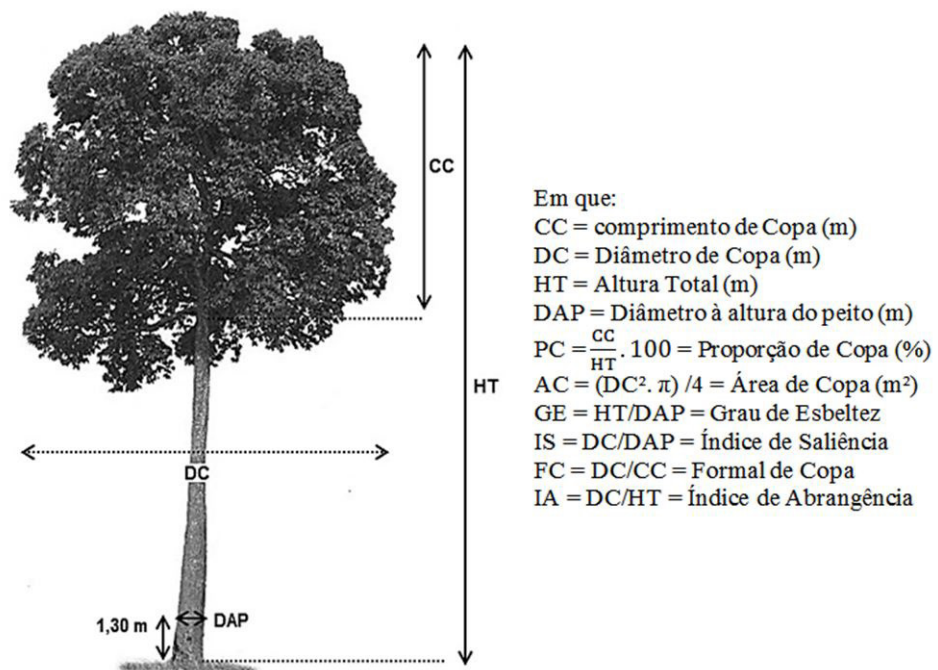

Figura 2. Modelo de uma árvore e suas relações morfométricas. Fonte: autores.

Figure 2. Model of a tree and its morphometric relationships. Source: authors.

Kolmogorov-Smirnov. Os dados para cada variável resposta foram avaliados pelos testes de Kruskal Wallis e qui-quadrado $\left(x^{2}\right)$, no caso de os pressupostos não serem atendidos.

As médias foram comparadas pelo teste de Games Howell a 5\% de probabilidade. As variáveis que não atenderam os pressupostos para análise de variância foram submetidas ao teste de Kruskal-Wallis e as médias foram comparadas pelo teste de Man-Withney à 5\% de probabilidade. Em todas as análises foi utilizado o software estatístico SAS.

\section{RESULTADOS E DISCUSSÕES}

Na Tabela 3 são apresentadas as variáveis analisadas aos seis anos de idade para o povoamento de Tectona grandis, desbastado nas intensidades de $0 \%$, 20\%, 30\% e 40\%. Foram observadas diferenças significativas para as variáveis morfométricas em função da intensidade do desbaste, com exceção da proporção de copa (PC) e índice de saliência (IS), o que indicou que a abertura de maiores espaços no povoamento de teca modificou a morfometria das árvores.

Autores como Tonini et al. (2009), Berger et al. (2002), Sanquetta et al. (2003) citam a influência direta da densidade na modificação das variáveis morfométricas e crescimento em povoamentos florestais.
Tabela 3. Teste não paramétrico para variáveis morfométricas. Altura comercial do fuste (HF), comprimento de copa (CC), proporção de copa (PC), diâmetro de copa (DC), grau de esbeltez (GE), índice de abrangência (IA), índice de saliência (IS) e formal de copa (FC), quatro anos após o desbaste.

Table 3. Non-parametric test for morphometric variables. Commercial shaft height $(\mathrm{HF})$, length of crow (CC), crow proportion (PC), crown diameter (DC) slenderness degree (GE), coverage index (AI), protrusion index (IS) and crown formal (FC), four years after thinning.

\begin{tabular}{cccc}
\hline Variável & GL & $\mathbf{X}^{2}$ & Significância \\
\hline DC & 3 & 14,421 & $0,002^{*}$ \\
HF & 3 & 19,577 & $0,000^{*}$ \\
\hline CC & 3 & 14,661 & $0,002^{*}$ \\
PC & 3 & 6,296 & $0,098^{\text {ns }}$ \\
\hline FC & 3 & 24,541 & $0,000^{*}$ \\
IA & 3 & 34,39 & $0,000^{*}$ \\
\hline IS & 3 & 4,741 & $0,192^{\text {ns }}$ \\
GE & 3 & 67,662 & $0,000^{*}$ \\
\hline
\end{tabular}

*significativo a $5 \%$ de probabilidade pelo teste de KruskalWallis. ${ }^{\text {ns }}$ não siginificativo a $5 \%$ de probabilidade pelo teste de Kruskal-Wallis.

Observa-se que as variáveis HF, CC, DC e PC não apresentaram diferença significativa em função da intensidade de desbaste $(20 \%, 30 \%$ e $40 \%)$, porém, diferem significativamente da testemunha $(0 \%)$ (Tabela 4). Verificou-se também que à medida que se aumenta a intensidade do desbaste, aumenta o ganho 
Tabela 4. Médias para altura comercial do fuste (HF), comprimento de copa (CC), proporção de copa (PC), diâmetro de copa (DC), grau de esbeltez (GE), índice de abrangência (IA), índice de saliência (IS) e formal de copa (FC), quatro anos após o desbaste.

Table 4. Averages for commercial shaft height (HF), length of crow (CC), crow proportion (PC), crown diameter (DC) slenderness degree (GE), coverage index (AI), protrusion index (IS) and crow formal (FC), four years after thinning.

\begin{tabular}{|c|c|c|c|c|c|c|c|c|}
\hline \multirow{2}{*}{ Desbaste } & HF & $\mathrm{CC}$ & DC & PC & GE & IA & IS & FC \\
\hline & \multicolumn{4}{|c|}{------------------m------------------ } & \multicolumn{4}{|c|}{---------------\%---------------- } \\
\hline $0 \%$ & $3,38 \mathrm{~b}$ & $8,88 \mathrm{~b}$ & $3,62 \mathrm{~b}$ & $64,82 \mathrm{~b}$ & $1,12 \mathrm{a}$ & $0,26 c$ & $28,15 \mathrm{a}$ & $0,38 \mathrm{~b}$ \\
\hline $20 \%$ & $3,93 \mathrm{a}$ & $8,87 \mathrm{ab}$ & $3,75 \mathrm{ab}$ & $70,83 \mathrm{a}$ & $0,93 \mathrm{~b}$ & $0,28 \mathrm{~b}$ & $26,48 \mathrm{a}$ & $0,41 \mathrm{ab}$ \\
\hline $30 \%$ & $4,03 \mathrm{a}$ & $9,77 \mathrm{a}$ & $4,03 \mathrm{a}$ & $72,01 \mathrm{a}$ & $0,91 \mathrm{c}$ & $0,30 \mathrm{~b}$ & $28,16 \mathrm{a}$ & $0,45 \mathrm{a}$ \\
\hline $40 \%$ & $4,25 \mathrm{a}$ & $10,19 a$ & $4,22 \mathrm{a}$ & $71,94 \mathrm{a}$ & $0,86 c$ & $0,32 \mathrm{a}$ & $26,78 \mathrm{a}$ & $0,42 \mathrm{a}$ \\
\hline
\end{tabular}

Médias seguidas de mesma letra na coluna não diferem estatisticamente entre si pelo teste de Man-Withney a $5 \%$ de probabilidade.

entre essas variáveis, indicando que está havendo incremento no povoamento após aplicação do desbaste. Esse resultado pode ser justificado pelo fato de que as práticas de desbaste promovem incremento e ganho em produtividade das árvores (Damásio et al., 2015) pois diminuem a competição entre elas e, com isso, aumentam o espaço vital, disponibilizando maior quantidade de recursos, principalmente água, luz e nutrientes.

De acordo com Nogueira et al. (2006) o desbaste, além de diminuir a competição entre as árvores, aumenta significativamente a taxa de crescimento das árvores remanescentes, uma vez que disponibiliza os fatores de produção para as árvores (dominantes e codominantes) mais eficientes na relação fotossíntese/respiração, aumentando a velocidade de crescimento dessas árvores.

Avaliando o efeito da intensidade do primeiro desbaste seletivo em um povoamento de Tectona grandis aos quatro anos de idade, Caldeira \& Oliveira (2008) observaram que as intensidades de desbaste de $20 \%, 40 \%$ e $50 \%$ afetaram a área basal e o volume, sendo inferiores ao da testemunha. Os mesmos autores ressaltam que o período de um ano pode ser insuficiente para que o incremento das árvores tratadas seja maior que o da testemunha.

Schneider (1993) relata que a resposta em crescimento é lenta, sendo que uma árvore pode levar até quatro anos para ampliar sua superfície foliar e radicular e, a partir daí, produzir um incremento significativo em diâmetro. Costa (2011) enfatiza que somente após quatro anos da realização dos desbastes se observam diferenciações nas áreas transversais (g), com maiores valores associados aos tratamentos desbastados.
O grau de esbeltez, ou relação H/DAP, é uma variável que caracteriza a estabilidade das árvores, e neste estudo, variou de 0,86 a 1,12 nas diferentes intensidades de desbaste. Quanto mais baixo o grau de esbeltez, mais estável é a árvore, quando esse índice ultrapassa $1 \%$ indica instabilidade (Tonini \& Arco-Verde, 2005). A diminuição do grau de esbeltez, com o aumento da altura das árvores, demostra que as mesmas estão crescendo mais em diâmetro do que em altura, sendo um fator positivo para sua estabilidade contra o vento, podendo mesmo servir como indicativo de desbastes em atraso, especialmente em povoamentos puros e equiâneos.

O DAP respondeu proporcionalmente às intensidades dos desbastes aplicados no povoamento de teca, estabelecendo-se a seguinte relação: quanto maior a sua intensidade, maior o ganho em DAP. Assim, os desbastes mais pesados (30\% e 40\%) promoveram maior ganho em diâmetro das árvores remanescente, apesar de que não houve diferença estatística significativa entre as diferentes intensidades de desbaste (Tabela 4).

Avaliando o desenvolvimento de teca em plantio homogêneo, Pelissari et al. (2013) observaram um decréscimo no incremento corrente anual em DAP até os cinco anos, seguido por um aumento aos seis anos, após a aplicação do desbaste, e aos nove anos, após o segundo desbaste. Pérez \& Kanninen (2005) indicaram desbastes aos cinco anos, para sítios de qualidade média, nas intensidades de $40 \%$ a $45 \%$, dependendo do objetivo e da rotação planejada.

Nogueira et al. (2006) salientam que o aumento do ingresso pode ser mais significativo se o desbaste for aplicado em uma idade mais apropriada, com uma intensidade maior, e se o povoamento remanescente 
não tiver árvores dominadas, ou seja, se o desbaste for seletivo, eliminando os piores indivíduos.

O índice de abrangência (IA), calculado pela relação (DC/HT) (Cunha \& Finger, 2013), apresentou diferença significativa entre T4 (40\%) e os demais tratamentos. Observou-se tendência de aumento desse índice com incremento em altura no T4, no qual houve remoção de $40 \%$ de área basal, ou seja, o aumento na altura da teca pós-desbaste é acompanhado, proporcionalmente, pelo aumento do diâmetro de copa.

A determinação do diâmetro de copa é de fundamental importância, pois permite realizar a projeção do espaço a ser reservado para uma árvore, de forma a garantir o seu bom desenvolvimento. De acordo com Roman et al. (2009), o conhecimento desse espaço, por sua vez, permite que se revele antecipadamente o número de indivíduos a serem selecionados e conduzidos até o final da rotação, quando se fixa um "diâmetro-objetivo".

O índice de saliência (IS), expresso pela relação (DC/DAP) (Cunha \& Finger, 2013), expressa quantas vezes o diâmetro de copa é maior que o DAP. No presente estudo, esse índice não apresentou diferença entre os tratamentos, indicando que a redução de $40 \%$ da área basal quatro anos após o primeiro desbaste, não influenciou na relação entre o crescimento do DAP e o diâmetro de copa. Esse índice apresentou uma média igual a 0,27 , sugerindo que a espécie estudada possui uma copa de 27 vezes o diâmetro de seu tronco a 1,3 m de altura.

A competição exerce efeito negativo no crescimento das árvores. De acordo com Santos et al. (2015), os tratamentos silviculturais e desbastes de condução são necessários para o manejo de povoamentos para obtenção de maior rendimento por hectare, independentemente de se tratar de espécies nativas ou exóticas. Certamente, aplicações de práticas de manejo, tais como estimativas de ciclos de corte (Schöngart, 2008) e a prescrição de tratamentos silviculturais específicos constituem ferramentas que podem contribuir com uma produção sustentável, do ponto de vista da produção de madeira (Cunha \& Finger, 2013).

Observa-se para o formal de copa (FC) que não houve diferença significativa entre os tratamentos que foram desbastados $(20 \%, 30 \%$ e $40 \%)$. Porém os tratamentos com maior redução de área basal (30\% e 40\%) diferiram significativamente em relação à testemunha (0\%). Esse resultado pode ser explicado pela liberação de um maior espaço na área da copa, ocasionada pelo desbaste, o que possibilitou um maior desenvolvimento do diâmetro (DC) e comprimento de copa (CC) desse povoamento, visto que o FC é a relação do (DC/CC).

Dessa forma, o desbaste disponibilizou o espaço aéreo para garantir um crescimento superior ao registrado para a testemunha, independentemente das intensidades utilizadas, até quatro anos após o tratamento.

Valores baixos de FC (menor que 1) indicam árvores com copas esbeltas, enquanto valores altos (maiores que 1) indicam copas achatadas (Orellana \& Koehler, 2008). Para a teca, os valores de FC foram inferiores a 0,45 , indicando que essa espécie apresenta uma copa mais esbelta do que achatada (Tabela 3 ).

Além dos parâmetros aqui apresentados, todos os outros fatores genéticos, biológicos e ambientais influenciam no crescimento das árvores, necessitando-se de estudos mais aprofundados. Vale ressaltar que estudos mais prolongados devem ser realizados para melhor entendimento do efeito do desbaste na morfometria da T. grandis.

\section{CONCLUSÃO}

A aplicação de desbastes em teca influenciou significativamente as variáveis morfométricas avaliadas, com exceção da proporção de copa (PC) e do índice de saliência (IS).

Quanto às diferentes intensidades de desbaste (20\%, 30\% e 40\%), não houve diferença significativa para as variáveis: altura comercial do fuste (HF), comprimento de copa (CC), diâmetro de copa (DC), proporção de copa (PC) e forma de copa (FC) da Tectona grandis até o quarto ano pós-desbaste.

\section{AGRADECIMENTOS}

Os autores agradecem à madeireira Mendes Ross Ltda. por disponibilizar a área de plantio de teca para esta pesquisa.

\section{STATUS DA SUBMISSÃO}

Recebido: 05 nov., 2014

Aceito: 21 abr., 2016 


\section{AUTOR(ES) PARA CORRESPONDÊNCIA}

\section{Luiz Fernandes Silva Dionisio}

Instituto de Ciências Agrarias, Universidade Federal Rural da Amazônia - UFRA, Avenida Presidente Tancredo Neves, No 2501 Bairro: Terra Firme, CEP 66077-830, Belém, PA, Brasil e-mail: fernandesluiz03@gmail.com

\section{REFERÊNCIAS}

Ângelo H, Silva VSM, Souza NA, Gatto AC. Aspectos financeiros da produção de teca no estado de Mato Grosso. Floresta 2009; 39(1): 23-32. http://dx.doi.org/10.5380/ rf.v39i1.13722.

Berger R, Schneider PR, Finger CAG, Haselein CR. Efeito do espaçamento e da adubação no crescimento de um clone de Eucalyptus saligna Smith. Ciência Florestal 2002; 2(2): 75-87. http://dx.doi.org/10.5902/198050981682.

Caldeira SF, Oliveira DLC. Desbaste seletivo em povoamentos de Tectona grandis com diferentes idades. Acta Amazonica 2008; 38(2): 223-228. http://dx.doi.org/10.1590/S004459672008000200005 .

Condé TM, Lima MLM, Lima EM No, Tonini H. Morfometria de quatro espécies florestais em sistemas agroflorestais no município de Porto Velho, Rondônia. Revista Agro@mbiente 2013; 7(1): 18-27.

Condé TM, Lima MLM, Lima Neto EM, Tonini H. Equações morfométricas das espécies Andiroba (Carapa guianensis Aubl) e Castanha-do-Brasil (Bertholletia excelsa H.B.K.) em sistemas agroflorestais no município de Porto Velho, Rondônia. Revista Eletrônica Ambiente Gestão e Desenvolvimento 2014; 4:1-21.

Costa KL. Crescimento de Tectona grandis (teca) em sistema silvicultural de talhadia composta em Minas Gerais [dissertação]. Lavras: Universidade Federal de Lavras; 2011.

Cunha TA, Finger CAG. Competição assimétrica e o incremento diamétrico de árvores individuais de Cedrela odorata L. na Amazônia ocidental. Acta Amazonica 2013; 43(1): 9-18. http://dx.doi.org/10.1590/S004459672013000100002 .

Damásio M, Silva FR, Santos AFA, Rondon RM No. Desbaste seletivo em um povoamento de Tectona grandis implantado em sistema de integração-lavoura-pecuáriafloresta. Biodiversidade 2015; 14(3): 74-83.

Durlo MA, Denardi L. Morfometria de Cabralea canjerana, em mata secundária nativa do Rio Grande do Sul. Ciência Florestal 1998; 8(1): 55-66. http://dx.doi. org/10.5902/19805098351

Durlo MA, Sutili FJ, Denardi L. Modelagem da copa de Cedrela fissilis Vellozo. Ciência Florestal 2004; 14(2): 7989. http://dx.doi.org/10.5902/198050981808.
Figueiredo EO. Reflorestamento com teca (Tectona grandis L.F.) no estado do Acre. Rio Branco: Embrapa Acre; 2001. (Documentos; no. 65).

Figueiredo EO, D’Oliveira MVN, Fearnside PM, Papa DA. Modelos para estimativa de volumes de árvores individuais pela morfometria da copa obtido com LIDAR. Cerne 2014; 20(4): 621-628. http://dx.doi.org/10.1590/0 1047760201420041693.

Figueiredo EO, Oliveira AD, Scolforo JRS. Análise econômica de povoamentos não desbastados de Tectona grandis L.f., na microrregião do rio Acre. Cerne 2005; 11(4): 342-353.

Medeiros RA, Paiva HN, Leite HG, Oliveira SN No, Vendrúscolo DGS, Silva FT. Análise silvicultural e econômica de plantios clonais e seminais de Tectona grandis l.f. em sistema taungya. Revista Árvore 2015; 39(5): 893-903. http://dx.doi.org/10.1590/0100-67622015000500012.

Miranda MC. Caracterização morfológica e avaliação do desenvolvimento inicial de clones de teca (Tectona grandis L.f.) [dissertação]. Cuiabá: Universidade Federal de Mato Grosso; 2013.

Motta JP, Oliveira JTS, Paes JB, Alves RC, Dambroz GBV. Resistência natural da madeira de Tectona grandis em ensaio de laboratório. Ciência Rural 2013; 43(8): 1393-1398. http://dx.doi.org/10.1590/S0103-84782013005000097.

Mourão M Jr, Xaud MR, Xaud HAM, Moura MA No, ArcoVerde MF, Pereira PRVS et al. Precipitação pluviométrica em áreas de transição savana-mata de Roraima: campos experimentais Serra da Prata e Confiança. Boa Vista: Embrapa Roraima; 2003. (Comunicado Técnico; no. 17).

Nogueira GS, Leite HG, Campos JCC, Takizawa FH, Couto L. Avaliação de um modelo de distribuição diamétrica ajustado para povoamentos de Tectona grandis submetidos a desbaste. Revista Árvore 2006; 30(3): 377-387. http:// dx.doi.org/10.1590/S0100-67622006000300008.

Orellana E, Koehler AB. Relações morfométricas de Ocotea odorifera (Vell.) Rohwer. Revista Acadêmica de Ciências Agrárias e Ambientais 2008; 6(2): 229-237.

Padoin V, Finger CAG. Relações entre as dimensões da copa e a altura das árvores dominantes em povoamentos de Pinus taeda L. Ciência Florestal 2010; 20(1): 95-105. http://dx.doi.org/10.5902/198050981764.

Pelissari AL, Caldeira SF, Drecher R. Desenvolvimento quantitativo e qualitativo de Tectona grandis L.f. em Mato Grosso. Floresta e Ambiente 2013; 20(3): 371-383.

Pérez D, Kanninen M. Stand growth scenarios for Tectona grandis plantations in Costa Rica. Forest Ecology and Management 2005; 210(1): 425-441. http://dx.doi. org/10.1016/j.foreco.2005.02.037.

Roman M, Bressan DA, Durlo MA. Variáveis morfométricas e relações interdimensionais para Cordia trichotoma (Vell) Arráb. ex Steud. Ciência Florestal 2009; 19(4): 473-480. http://dx.doi.org/10.5902/19805098901. 
Sanquetta CR, Arce JE, Mello AA, Silva EQ, Barth N Fo, Matoski SLS. Produção de Madeira livre de nós em povoamentos de Pinus taeda em função da densidade de plantio. Cerne 2003; 9(2): 129-140.

Sanquetta CR, Behling A, Corte APD, Fernandes AC, Beckert SM, Simon AA. Equações para estimativa do diâmetro de copa para Acácia-negra. Floresta e Ambiente 2014; 21(2): 192-205. http://dx.doi.org/10.4322/floram.2014.018.

Santos AT, Mattos PP, Braz EM, Rosot NC. Determinação da época de desbaste pela análise dendrocronológica e morfométrica de Ocotea porosa (nees \& mart.) Barroso em povoamento não manejado. Ciência Florestal 2015; 25(3): 699-709. http://dx.doi.org/10.5902/1980509819620.

Santos HG, Jacomine PKT, Anjos LHC, Oliveira VA, Oliveira JB, Coelho MR et al., editores. Sistema brasileiro de classificação de solos. 2. ed. Rio de Janeiro: Embrapa Solos; 2006.

Santos MJC, Santos FR, Ribeiro MJB. Parâmetros interdimensionais de clones de eucalipto em sistema silvipastoril na região do semiárido. Cadernos de Agroecologia 2014; 9(4): 1-11.

Schneider PR. Introdução ao manejo florestal. Santa Maria: CEPEF/FATEC; 1993.348 p.

Schöngart J. Growth-Oriented Logging (GOL): a new concept towards sustainable forest management in Central Amazonian várzea floodplains. Forest Ecology and Management 2008; 256(1): 46-58. http://dx.doi. org/10.1016/j.foreco.2008.03.037.
Schuhli GS, Paludzyszyn E Fo. O cenário nacional da silvicultura de teca (Tectona grandis L. f.) e perspectivas de melhoramento. Pesquisa Florestal Brasileira 2010; 30(63): 217-230. http://dx.doi.org/10.4336/2010.pfb.30.63.217.

Silveira BD, Floriano EP, Nakajima NY, Hosokawa RT, Rosot NC, Gracioli CR. Relação da morfometria e competição com o crescimento de Trichilia claussenii em um fragmento de floresta semidecidual, RS. Floresta 2015; 45(2): 373-382. http://dx.doi.org/10.5380/rf.v45i2.35164.

Tonini H, Arco-Verde MF. Morfologia da copa para avaliar o espaço vital de quatro espécies nativas da Amazônia. Pesquisa Agropecuária Brasileira 2005; 40(7): 633-638. http://dx.doi.org/10.1590/S0100-204X2005000700002.

Tonini H, Costa MCG, Schwengber LAM. Crescimento da teca (Tectona grandis) em reflorestamento na Amazônia setentrional. Pesquisa Florestal Brasileira 2009; 59: 5-14.

Tonini H, Kaminski PE, Costa P. Relação da produção de sementes de castanha-do-brasil com características morfométricas da copa e índices de competição. Pesquisa Agropecuária Brasileira 2008; 43(11): 1509-1516. http:// dx.doi.org/10.1590/S0100-204X2008001100009.

Weber VP, Finger CAG, Fleig FD, Souza CAM, Munareto FF, Silva LTM. Diretrizes para desbaste de Pinus taeda L. Em função da altura dominante. Ciência Florestal 2013; 23(1): 193-201. http://dx.doi.org/10.5902/198050988453.

Wink C, Monteiro JS, Reinert DJ, Liberalesso E. Parâmetros da copa e a sua relação om diâmetro e altura das árvores de eucalipto em diferentes idades. Scientia Forestalis 2012; 40(93): 57-67. 